\title{
SIGNIFICADO PROGNÓSTICO DAS METÁSTASES NOS LINFONODOS REGIONAIS DO ADENOCARCINOMA COLO-RETAL ${ }^{1}$
}

\author{
Sansom Henrique Bromberg ${ }^{2}$ \\ Elci Barreto ${ }^{3}$ \\ José Eduardo Cajado Moncau ${ }^{4}$ \\ Saul Goldenberg ${ }^{5}$
}

\begin{abstract}
BROMBERG, S.H.; BARRETO, E; MONCAU, J.E.C.; GOLDENBERG, S. - Significado prognóstico das metástases nos linfonodos regionais do adenocarcinoma colo-retal. Acta Cir. Bras., 12(1):67-73, 1997.

RESUMO: Trezentos e vinte doentes com adenocarcinoma colo-retal submetidos a ressecção curativa foram estudados com o intuito de verificar, nas peças ressecadas, o comprometimento ou não dos linfonodos, o númcro dos acometidos e a área ocupada pela neoplasia. Chamou-se de linfonodos invadidos aqueles com raros focos de células neoplásicas presentes, que não interferiam na estrutura do nodo e de destruídos àqueles que apresentavam sua estrutura $\mathrm{em}$ grande parte ou totalmente ocupada pelo tumor. Os linfonodos positivos foram agrupados em subgrupos contendo 1 a 4 e em mais de 4.120 (38,6\%) doentes apresentaram linfonodos comprometidos, dos quais $24,2 \%$ eram invadidos e $75,8 \%$ destruidos. Portadores de linfonodos livres apresentaram sobrevivência de cinco anos de $71,7 \%$; a sobrevivência de doentes com nodos invadidos - $58,6 \%$ - foi significantemente maior que a dos com nodos destruidos - $29,7 \%$, mas não diferiu estatisticamente da apresentada pelos portadores de linfonodos livres. Doentes com mais de 4 linfonodos comprometidos sobreviveram significantemente menos $(20,0 \%)$ que aqueles com 1 a 4 linfonodos acometidos $(43,5 \%)$. Entre os portadores de linfonodos invadidos a sobrevivência de 5 anos foi a mesma para os com 1 a 4 ou mais de 4 linfonodos invadidos. Isto não aconteceu com portadores de linfonodos destruidos, onde os com 1 a 4 sobreviveram em cerca de $37,7 \%$ e os com mais de $4,13,3 \%$. Faz-se necessário identificar adequadamente a área ocupada pela neoplasia no linfonodo, pois o comportamento biológico do tipo invadido diferiu substancialmente do destruido. $\mathrm{O}$ melhor prognóstico dos portadores de linfonodos invadidos pode explicar a sobrevivência aparentemente paradoxal observada em alguns doentes estadiados na classe $\mathrm{C}$ de DUKES.
\end{abstract}

DESCRITORES: Carcinoma colo-retal. Prognóstico. Linfonodos.

\section{INTRODUÇÃO}

Os parâmetros mais utilizados no prognóstico do adenocarcinoma colo-retal extirpado são fornecidos pelo estádio patológico. O nível de penetração neoplásica na parede intestinal e o comprometimento ou não dos linfonodos, segundo a classificação de DUKES, vem sendo utilizados com sucesso há mais de meio século. (DUKES, 1932) ${ }^{10}$

GABRIEL, DUKES e BUSSEY (1935) ${ }^{16} \mathrm{e}$ DUKES e BUSSEY (1958)" ampliaram a participa- ção dos linfonodos comprometidos no prognóstico ao estabelecerem que o seu número e o nível de sua localização exerce influência na sobrevida. Trabalhos posteriores confirmaram suas observações. (SPRATT e SPJUT, 1967) ${ }^{34}$; COPELAND, MILLER e JONES, $1968)^{\mathrm{x}}$

No entanto, o comprometimento dos linfonodos pela neoplasia não é homogêneo. Doentes com pequenas áreas de seus linfonodos ocupadas por células neoplásicas (BROMBERG, 1984) ${ }^{3}$, portadores de micrometastases (HUVOS, HUTTER e BERG, 1971) ${ }^{19}$ ou com metástases ocultas (CUTAIT, 1987) ${ }^{9}$, apresen-

1. Parte da Tese de Doutorado apresentada no Curso de Pós-Graduação em Técnica Operatória e Cirurgia Experimental da UNIFESP-EPM. Trabalho realizado no Serviço de Gastroenterologia Cirúrgica (Diretor: Dr. Antonio Cláudio de Godoi) e no Serviço de Anatomia Patológica do Hospital do Servidor Público Estadual de São Paulo - Francisco Morato de Oliveira (HSPE-FMO)

2. Doutor pelo Curso de Pós-Graduação em Técnica Operatória e Cirurgia Experimental da Universidade Federal de S.Paulo - Escola Paulista de Medicina (UNIFESP - EPM)

3. Diretora do Serviço de Anatomia Patológica do HSPE-FMO.

4. Prof. Adjunto do Disciplina de Biocstatística da UNIFESP - EPM

5. Prof. Titular da Disciplina de Técnica Operatória e Cirurgia Experimental do Departamento de Cirurgia da UNIFESP EPM. Orientador da Tese. 
taram sobrevivência próxima à de doentes com linfonodos livres e muito superior a dos que abrigam linfonodos estruturalmente destruídos pela neoplasia.

Para JASS $(1990)^{20}$, o papel dos linfonodos no prognóstico seria melhor avaliado se, além do local e do número de linfonodos acometidos, fosse também considerado outros parâmetros tais como, a invasão da cápsula e a área do linfonodo ocupada pelas células neoplásicas, variáveis essas pouco lembradas pelos estudiosos.

O intuito do presente trabalho é estudar o comprometimento neoplásico dos linfonodos de portadores de adenocarcinoma colo-retal, levando em consideração seu número e a área acometida pela doença, relacionando esses parâmetros com o prognóstico..

\section{MÉTODO}

Analisou-se os dados de 320 doentes com adenocarcinoma colo-retal submetidos a ressecção considerada curativa no período de 1964 a 1980 no Serviço de Gastroenterologia Cirúrgica do HSPEFMO.

Foram excluídos do estudo os enfermos com seguimento inferior a cinco anos, os que faleceram nos dois primeiros meses de pós-operatório e os falecidos em período inferior a cinco anos por causas não relacionadas a neoplasia.

O acompanhamento clínico desses doentes foi feito pela análise de seus prontuários e mediante contatos pessoais com os mesmos ou seus aparentados.

Dos 320 doentes, $300(93,75 \%)$ eram brancos, $10(3,12 \%)$ eram pardos, $6(1,88 \%)$ amarelos e 4 $(1,25 \%)$ eram negros.

A idade dos mesmos variou de 28 a 86 anos, com mediana de 58 anos.

Das neoplasias, $135(42,2 \%)$ situavam-se no reto, $112(35,0 \%)$ no colo esquerdo e $73(22,8 \%)$ no colo direito.

Foram efetuados os seguintes procedimentos operatórios: 64 colectomias direitas, 97 colectomias esquerdas, 14 sigmoidectomias, 96 amputações abdomino-perineais do reto, 21 reto-colectomias abdominais, 15 reto-colectomias abdomino-perineais, 16 transversectomias, 4 colectomias totais e 3 proctocolectomias totais.

$\mathrm{O}$ estudo anátomo-patológico foi realizado por uma patologista do mesmo hospital que desconhecia a evolução clínica dos doentes. Após a inspecção macroscópica da peça fixada em formol a $10 \%$, os linfonodos eram dissecados da gordura mesentérica a partir de seu pedículo vascular, seccionados longitudinalmente em sua porção mediana e processados para estudo com hematoxilina e eosina, sendo as lâminas interpretadas sob objetivas de pequeno ( 40 $\mathrm{x})$, médio $(100 \mathrm{x})$ e grande aumento $(400 \mathrm{x})$.

Os linfonodos foram estudados quanto à presença ou não de comprometimento neoplásico e quanto ao número de acometidos. Na ausência de metástases, os linfonodos foram chamados de livres e na sua presença de comprometidos. Estes foram distribuidos em dois grupos:

a) invadidos - quando se constatou estar a maioria dos linfonodos livres, havendo focos de células neoplásicas em alguns deles, sem interferir na sua estrutura habitual e nunca atingindo a cápsula do linfonodo

b) destruídos - quando a maioria deles se mostrava tomada pela neoplasia, muitos com a cápsula acometida, com sua estrutura habitual substituida total ou em sua maior parte por tecido neoplásico.

Caracterizou-se três grupos de enfermos de acordo com o número de nodos linfáticos acometidos: os com linfonodos livres, os com 1 a 4 comprometidos e aqueles com 5 ou mais nodos comprometidos, como preconizado por JASS e MORSON (1987) ${ }^{21}$.

Em nove das peças cirúrgicas ressecadas não foram encontrados linfonodos.

Para análise dos resultados foram utilizados testes não paramétricos: qui-quadrado, partição do quii-quadrado e teste exato de FISHER A sobrevida cumulativa de dez anos foi analisada pelo método de KAPLAN-MEIER, cujo programa utiliza o teste de COX-MANTEL (produto momento) e o de WILCOXON (generalizado), fornecido pelo Ludwig Institute for Cancer Research, de S. Paulo. (COCHRAN, 1954 ${ }^{6}$; SIEGEL, 1975 ${ }^{33}$, LEE, 1980) $)^{23}$

Em todos os testes fixou-se em 0,05 ou $5 \%$ o nível para rejeição da hipótese de nulidade. Os valores significantes foram assinalados com asterístico $(*)$

\section{RESULTADOS}

A sobrevida de cinco anos, independente de qualquer variável, foi de $58,1 \%$.

Os linfonodos mostraram-se livres de comprometimento neoplásico em $191(61,4 \%)$ dos espécimes ressecados e acometidos em $120(38,6 \%)$ deles.

A presença de neoplasia no linfonodo influiu desfavoravelmente na sobrevivência de seus portadores. (Tabela I) 
Tabela I - Grau de comprometimento dos linfonodos e sobrevida de cinco anos de 311 enfermos com câncer coloretal

\begin{tabular}{ccccc}
\hline Linfonodos & $\geq 5 \mathrm{a}$ & $\%$ & $<5 \mathrm{a}$ & Total \\
\hline livres & 137 & 71,7 & 54 & 191 \\
comprometidos & 44 & 36,7 & 76 & 120 \\
\hline Total & 181 & 130 & 311 & \\
\hline
\end{tabular}

$\mathrm{X}^{2}$ cal $=37,23 * \mathrm{X}^{2}$ crit $=3,84$

A sobrevivência aos 10 anos, estimada pelo método de KAPLAN-MEIER , foi de $63,8 \pm 3,5 \%$ para portadores de linfonodos livres e de $31,5 \pm 4,3 \%$ para os com linfonodos comprometidos. $(p<0,0001)$

Ao avaliar-se os linfonodos comprometidos notou-se predomínio dos destruídos e do contingente de 1 a 4 nodos permeados pelo tumor. (Tabela II)

Tabela II - Tipo de comprometimento e número de linfonodos acometidos em 120 espécimes de câncer coloretal

\begin{tabular}{lcc}
\hline Comprometimento & Freqüencia & $\%$ \\
\hline tipo & 29 & 24,2 \\
invadidos & 91 & 75.8 \\
destruídos & 85 & 70,8 \\
numero & 35 & 29,2 \\
! a 4 & 120 & 100,0 \\
\hline e+ & & \\
\hline
\end{tabular}

A sobrevivência dos doentes com linfonodos invadidos mostrou-se significantemente maior que a apresentada por aqueles com linfonodos destruídos e próxima daquela apresentada pelos doentes sem comprometimento neoplásico de seus linfonodos. (Tabela III)

Tabela III - Grau de acometimento dos linfonodos e sobrevida de cinco anos em 311 doentes com câncer coloretal

\begin{tabular}{lcccc}
\hline Linfonodos & $\geq 5 \mathrm{a}$ & $\%$ & $<5 \mathrm{a}$ & Total \\
\hline (a) livres & 137 & 71,7 & 54 & 191 \\
(b) invadidos & 17 & 58,6 & 12 & 29 \\
(c) destruidos & 27 & 29,7 & 64 & 91 \\
\hline Total & 181 & 130 & 311 & \\
\hline
\end{tabular}

$\mathrm{X}^{2}$ cal $=44,81 * \mathrm{X}^{2}$ crit $=5,99$

$$
\begin{gathered}
\text { Partição do qui-quadrado } \\
\mathrm{X}^{2} \text { crit }=5,02
\end{gathered}
$$

A sobrevida estimada de 10 anos para portadores de linfonodos invadidos foi de $49,01,0 \%$ e de 25,8 $4,6 \%$ para os com linfonodos destruídos $(p<0,0001)$.

$\mathrm{O}$ número de linfonodos acometidos pela neoplasia correlacionou-se com a sobrevida. Doentes com mais de cinco linfonodos comprometidos apresentaram menor tempo de sobrevivência que os com 1 a 4. (Tabela IV)

Tabela IV - Sobrevida de cinco anos de 120 portadores de neoplasias colo-retais segundo o número de linfonodos comprometidos

\begin{tabular}{lcccc}
\hline Comprometimento & $\geq 5 \mathrm{a}$ & $\%$ & $<5 \mathrm{a}$ & Total \\
\hline I a 4 & 37 & 43,5 & 48 & 85 \\
$5 \mathrm{e}+$ & 7 & 20,0 & 28 & 35 \\
\hline Total & 44 & 76 & 120 & \\
\hline
\end{tabular}

$\mathrm{X}^{2}$ cal $=5,91 * \mathrm{X}^{2}$ crit $=3,84$

Portadores de 1 a 4 linfonodos comprometidos apresentaram sobrevivência estimada de 10 anos de $36,1 \pm 5,4 \%$ e os com mais de 5 linfonodos comprometidos $20,0 \pm 6,7 \%(p<0,0041)$.

No tocante ao número de linfonodos invadidos, não foi constatada diferença significativa na sobrevivência de cinco anos entre o grupo com 1 a 4 e aquele com mais de 5 linfonodos acometidos da mesma forma (Tabela V).

Tabela V - Sobrevida de cinco anos de 29 enfermos com câncer colo-retal segundo o número de linfonodos invadidos

\begin{tabular}{lcccc}
\hline Linfonodos & ${ }^{3} 5 a$ & $\%$ & $<5 a$ & Total \\
\hline invadidos $\left(\begin{array}{llll}1 & \mathrm{a} & 4\end{array}\right)$ & 14 & 58,3 & 10 & 24 \\
invadidos $(5 \mathrm{c}+4)$ & 3 & 60,0 & 2 & 5 \\
\hline Total & 17 & 12 & 29 & \\
\hline
\end{tabular}

- Teste de FISHER -

$p=0,6705$ ou $67,05 \%$

Já para os doentes com nodos linfáticos destruídos, a sobrevivência mostrou-se significantemente pior para aqueles com cinco ou mais linfonodos acometidos (Tabela VI).

Tabela VI - Sobrevida de cinco anos de 91 enfermos com câncer colo-retal, segundo o número de linfonodos destruídos

\begin{tabular}{lcccc}
\hline Número & $\geq 5 \mathrm{a}$ & $\%$ & $<5 \mathrm{a}$ & Total \\
\hline destruídos $\left(\begin{array}{llll}1 & \mathrm{a} & 4\end{array}\right)$ & 23 & 37,7 & 38 & 61 \\
destruídos $\left(\begin{array}{ll}5 & \mathrm{e}+\end{array}\right)$ & 4 & 13,3 & 26 & 30 \\
\hline Total & 27 & 64 & 91 & \\
\hline
\end{tabular}

$\mathrm{X}^{2} \cdot \mathrm{cal}=5,72 * \mathrm{X}^{2}$ crit $=3,84$ 


\section{DISCUSSÃO}

Os indices medios de sobrevivência de cinco anos relatados na literatura mostram-se similares. em diferentes populações.

COPELAND e col. $(1968)^{8}$ dos Estados Unidos, OHMAN $(1985)^{26}$ da Suécia e BRANDÃO, SOBRINHO-SIMÕES e SERRÃO (1985) de Portugal, comunicaram índices de sobrevivencia média quase idênticos: $48,1 \%, 49 \%$ e $48,8 \%$. Na Alemanha cla foi de $41,2 \pm 3,9 \%$ (SCHMITZ-MOORMAN, HIMMELMANN, BAUM e NILES, 1987) $)^{31}$ e no Brasil, BRENNER $(1990)^{2}$ relatou sobrevida média de $45 \%$.

Neste trabalho ela foi de $58,1 \%$, valor superior à media daqueles relatos, talvez influenciada por um contingente maior de neoplasias classificadas como DUKES A.

A importância de se avaliar a extensão do acometimento linfático pelo carcinoma, obriga ao estudo de todos os linfonodos que drenam o segmento intestinal acometido pela neoplasia. Assim, o isolamento dos mesmos da gordura mesentérica deve ser exaustivo c cuidadoso, com a maioria dos nodos linfáticos comprometidos sendo encontrados próximos a parede intestinal onde se assesta o tumor (peri-cólicos). Linfonodos menores de $5 \mathrm{~mm}$ são freqüentemente atingidos pela neoplasia. (HERRERA-ORNELAS, JUSTINIANO, PETRELLI, MITTELMANN, 1987) $)^{17}$

Alguns patologistas advogam o emprego da técnica de clareamento da gordura mesentérica, afirmando que com ela conseguem aumentar o número de linfonodos examinados e também o dos comprometidos (SCHMITZ - MOORMAN e col., 1987; ${ }^{3 !}$ SCOTT E GRACE, 198932; HERRERA ORNELAS e col., 1987 17 , embora não alterem o estádio de DUKES (CAWTHORN, GIBBS, MARKS, 1986'). Outros, no entanto, mostram-se satisfeitos com o método clássico de seu isolamento (JASS e MORSON, 1987) ${ }^{21}$, por não estarem convencidos que o maior tempo dispendido, o maior esforço e o maior custo compensem os resultados obtidos.

O porcentual de linfonodos comprometidos pela neoplasia maligna aumenta com maior número de secções dos linfonodos (WILKINSON, HAUSE, $1974^{37}$ ) e com o emprego da imunohistoquímica (CUTAIT, 1987) 9 .

Neste estudo foram empregados métodos clássicos de isolamento, não sendo encontrados nodos linfáticos em nove das peças examinadas.

A maioria dos estudiosos indica como variável mais importante no prognóstico do câncer colo-retal a presença ou não de comprometimento dos linfonodos. Sabe-se que à medida que aumenta o número dos nodos linfáticos comprometidos diminui a sobrevivência.

DUKES e BUSSEY (1958)" assinalaram uma sobrevida de cinco anos de $83,7 \%$ para os enfermos sem acometimento dos linfonodos, que, quando presente, determinou uma sobrevivencia de $32 \%$. Para SPRATT e SPJUT $(1967)^{34}$ estes dados foram respectivamente de $47 \%$ e $20 \%$; para SHCMITZMOORMANN e col. $(1987)^{31}$ de $72,8 \%$ e $30,9 \%$ e no presente estudo de $71,7 \%$ e $36,7 \%$.

Quando um linfonodo estava comprometido a sobrevivência caiu para $63 \%$ e frente ao comprometimento de 2 a 5 nodos linfáticos reduziu-se a $36,1 \%$ no estudo de DUKES e BUSSEY (1958) ${ }^{11}$. Na vivencia de MURRAY, ARENO, DUTTON e HAMPSON $(1975)^{25}$, quando um linfonodo estava comprometido, a sobrevida foi de $58 \%$; com dois nodos comprometidos foi de $53,8 \%$, com três foi de $41,7 \%$ e com mais de quatro, $35 \%$. Sobrevida similar foi relatada por WILSON e BEAHRS, em 1976, para pacientes com mais de 4 linfonodos comprometidos $-30,1 \pm 5,7 \%{ }^{36}$

Desde que mais de 5 nodos linfáticos estejam envolvidos pela neoplasia cresce o número de linfonodos distantes comprometidos. (WOLMARK, FISHER, WIEAND e FISHER, 198438; PAYNE, $1989^{27}$ )

Estes mesmos autores concluiram ser o risco relativo de óbito 2,2 vezes maior para doentes com mais de quatro linfonodos positivos quando compa rados àqueles com um a quatro nodos linfáticos envolvidos pela neoplasia (WOLMARK, FISHER, WIEAND, 1986). ${ }^{39}$

Para COPELAND e col. $(1968)^{\circ}$ somente $10 \%$ dos enfermos com cinco ou mais nodos linfáticos comprometidos atingiram sobrevivência de cinco anos, enquanto outros não encontraram sobreviventes de cinco anos com este contingente de linfonodos acometidos. (SCHMITZ-MOORMANN e col., 1987) ${ }^{31}$

JASS (1990) ${ }^{20}$ referiu sobrevivência corrigida de 5 e 10 anos igual a $44 \%$ e $39 \%$ respectivamente para doentes com 1 a 4 nodos linfáticos comprometidos; quando mais de 5 nodos mostraram-se acometidos a sobrevida reduziu-se para $13 \%$ e $6 \%$ respectivamente.

Neste trabalho, portadores de 1 a 4 linfonodos comprometidos apresentaram sobrevivência de cinco anos de $43,5 \%$, que naqueles com cinco ou mais nodos linfáticos caiu para $20 \%$. A sobrevida estimada de dez anos foi de $36,1 \pm 5,4 \%$ e $20,0 \pm 6,7 \%$, respectivamente.

Portadores de múltiplos linfonodos acometidos pela neoplasia mais facilmente apresentam focos microscópicos da doença disseminados pelo duto torácico, com freqüentes metastases ao nivel do fïgado. 
Estes resultados confirmam ser o número de linfonodos comprometidos variável prognóstica de maior importância, por traduzir estádio mais avançado da doença.

Ao registrarem o comprometimento nodal, a maioria dos estudiosos não faz menção à extensão da área acometida e tampouco à integridade da cápsula do linfonodo.

Estudando carcinomas mamários, HUVOS e col $(1971)^{19}$ definiram micrometástases como sendo lesões menores que $2 \mathrm{~mm}$, sendo as maiores denominadas de macro-metástases. Em seu estudo, constataram que enfermas com micrometástases $\mathrm{em}$ linfonodos axilares do grupo inferior apresentaram na prática, a mesma sobrevivência que as sem metástases. Outros estudiosos obtiveram os mesmos resultados (ROSEN, SAIGO, BRAUN, WEAHERS, FRACCHIA e KINNE, 1981) ${ }^{30}$. Também foi observado que doentes com micrometástases subcapsulares apresentaram sobrevivência maior que aquelas com micrometástases detetadas na intimidade do linfonodo. (FISHER, SWAMIDOS, LEE, ROCKETTE, REDMOND e FISHER, 1978) ${ }^{15}$.

Em estudo de câncer colo-retal, CUTAIT (1987) ${ }^{9}$ evidenciou a presença de metástases ocultas em $25,5 \%$ dos linfonodos de espécimes catalogados no estádio A e B de DUKES, ao detetar nos mesmos a presença de CEA pela imunoperoxidase; a maioria dessas metástases ocultas situava-se em nível subcapsular. Ao correlacionar o prognóstico desses doentes com os livres de metástases, não encontrou diferença na evolução dos mesmos, justificando tal fato pelo caráter incipiente da invasão nodal.

Às mesmas conclusões chegaram JEFFERS, O'DOWD, MULCAHY, STAGG, O'DONOGHUE e TONER $(1994)^{22}$ ao empregarem citoceratina no reconhecimento de metastases nodais em neoplasias no estádio B de DUKES. A sobrevivência desses doentes $(25,0 \%)$ não diferiu estatisticamente da dos portadores de linfonodos livres $-47,0 \%$ ao término de cinco anos.

Os linfonodos próximos às neoplasias colo-retais freqüentemente se apresentam tensos, elásticos e esféricos devido ao notável aumento de seus componentes celulares. (PHIL, NAIRN, HUGHES, CUTHBERTSON, ROLLO,1980) ${ }^{29}$. Geralmente não abrigam células neoplásicas, que quando presentes, ocupam pequena área do nodo.

A presença de linfonodos destruídos é mais comum em pequenos linfonodos - menores que $5 \mathrm{~mm}$ - e as micrometástases em linfonodos maiores de $5 \mathrm{~mm}$ (HERRERA-ORNELAS e col., 1987). ${ }^{17}$

No carcinoma mamário constata-se que ao atingirem a cápsula do linfonodo, as células neoplásicas infiltram o tecido adiposo e os vasos linfáticos adjacentes ao nodo, adquirindo assim a neoplasia maior extensão e conseqüentemente pior prognóstico. (MAMBO e GALLAGHER, 1977) ${ }^{24}$

A extensão das metástases até a cápsula dos linfonodos associa-se também a um maior número de nodos comprometidos; o comprometimento e rutura da cápsula nodal constituem-se em marcadores de recidiva e sinal de mau prognóstico. (FISHER, SAFFER, FISHER, 1973) $)^{13}$

Conforme visto na Tabela $\mathrm{V}$, doentes com linfonodos invadidos apresentaram sobrevida de cinco anos próxima a daqueles com linfonodos livres $58,6 \%$ e $71,7 \%$ - sem que esta diferença fosse significante; já os portadores de linfonodos destruídos pela neoplasia apresentaram sobrevivência muito inferior - $29,7 \%$. Obtivemos resultados similares quando do estudo de carcinomas gástricos (BROMBERG, 1984) $)^{3}$

A sobrevivência estimada desses doentes aos dez. anos registra dados similares; naqueles com linfonodos livres ela é estimada em 53,8\% 3,5\%, nos com linfonodos invadidos em $49,0 \% \quad 10 \%$ e nos com linfonodos destruídos em $25,8 \% 4,6 \%$.

Seguramente, parte dos linfonodos por nós catalogados como invadidos obedeciam o critério de micrometástase, permitindo supor para todo o grupo com linfonodos invadidos um comportamento biológico favorável.

Sabe-se que as células neoplásicas penetram nos linfonodos pelos seios subcapsulares e daí progridem para os seios para-corticais e medulares (FISHER, FISHER, SAFFER, 1977) ${ }^{14}$. O tempo parece ser uma das variáveis que regulam esta progressão, que se faz rapidamente em alguns doentes, mas podendo ser extremamente lenta em outros, aparentemente traduzindo aspectos da agressividade biológica do tumor. $(\text { CADY, 1984) })^{4}$

Parece-nos atrativa a hipótese de células neoplásicas adentrarem a linfonodos cujos componentes celulares estejam menos aptos a se defenderem dessa invasão. Assim, metástases linfáticas poderiam estar condicionadas à densidade linfocitária do nodo (Van de VELDE, CARR, 1977) ${ }^{35}$ e a atividade citotóxica de celulas especiais, capazes de exercer ação inibitória sobre as células neoplásicas. (FISHER, SAFFER e FISHER, $1972^{12}$; PIHL, NAIRN, NIND, MULLER, HUGHES, CUTHBERTSON, ROLLO, $1976^{28}$; HOON, KORN, COCHRAN, 1987) 18

À medida que as células neoplásicas substituissem a estrutura habitual dos linfonodos, gradativamente impossibilitariam eventuais respostas defensivas de seus componentes (HOON e col. 1987) ${ }^{18}$

Quando a estrutura do linfonodo é substituída em grande parte por metástases, a perfusão linfática pela polpa linfonodal diminui ou é abolida e as 
células tumorais são carreadas para outros nodos vizinhos. Tal obstrução favorece a disseminação retrógrada, atingindo assim linfonodos mais distantes do tumor primário. (COHEN, TREMITERRA, CANDELA, THALER, SIGURDSON, 1991)?

$\mathrm{O}$ número de linfonodos invadidos não interferiu na sobrevivência dos doentes, o que não aconteceu quando os nodos linfáticos estavam destruidos pela neoplasia. Assim, a presença de 4 ou mais nodos positivos só acarretou pior prognóstico quando os mesmos eram do tipo destruidos.

A presença de neoplasia no linfonodo não é indicativa sistemática de pior prognóstico ou de menor sobrevida, não implicando necessariamente em disseminação da doença, como se constatou em mais de $1 / 3$ dos nossos doentes com neoplasias no estádio $\mathrm{C}$ de DUKES.
$\mathrm{O}$ estudo dos linfonodos com pequenos focos neoplásicos deve ser estimulado. Esses estudos podem, eventualmente, explicar algumas das interações dos componentes celulares do nodo com as células neoplásicas.

\section{CONCLUSÕES}

Em neoplasias colo-retais, os linfonodos devem ser estudados não só quanto a presença ou não de células neoplásicas e quanto ao número de comprometidos, mas também quanto a área ocupada pelas células neoplásicas, visto ser o comportamento biológico dos linfonodos invadidos substancialmente diferente dos destruídos.

BROMBERG, S.H.; BARRETO, E.; MONCAU, J.E.C.; GOLDENBERG, S. - Prognostic significance of regiona! lymph node metastases in colorectal carcinoma. Acta Cir. Bras., 12(1):67-73, 1997.

SUMMARY: 320 patients submitted to curative ressection for colorectal carcinoma was analysed with reference to involved lymph nodes, with the number of their and with the area taken by the tumor. The involved lymph nodes were named invaded when neoplastic nidus cells were present in some of them, with their habitual structure present and destroyed when there was a great extent of node replacement by neoplastic cells, and with many of them with capsular invasion. The extent of nodal metastases were graded from 1 to 4 and more than 4 nodes involved. $120(38,6 \%$ ) patients had positive nodes, with $24.2 \%$ of invaded type and $75,8 \%$ of destroyed type. The five-year survival rate of 191 patients with free lymph nodes was $71,7 \%$. The five-year survival rate of patients with invaded lymph nodes $(58,6 \%)$ was better than those of patients with destroyed lymph nodes - 29,7\% -and showed a similar behaviour to patients with free lymph nodes $(p=0,6705)$. Those patients showing 5 or more involved lymph nodes have a worse five-year survival rate $(20 \%)$ than patients with 1 to 4 positive nodes $(43,5 \%)$. The number of invaded lymph nodes don't was related to survival-rate but patients with 1 to 4 destroyed lymph nodes havea better 5-year survival $(37,4 \%)$ than patients with more of 4 nodes destroyed $(13,3 \%)$ The best prognosis of invaded lymph nodes can explain the paradoxical survival of several patients in the DUKES'C class.

SUBJECT HEADINGS: Colorectal cancer. Prognosis. Lymph node.

\section{REFERÊNCIAS}

1. BRANDÃO, O.; SOBRINHO-SIMÕES, M.A.; SERRÃO, D. - Prognosis in colorectal carcinoma: a reassessment of the pathologist's role. Path. Res. Pract.. 180:50610,1985

2. BRENNER, S. - Adenocarcinomas colorretais. Análise dos resultados do tratamento cirúrgico em 608 doentes. Curitiba, 1990. 149 pg. (TESE - Professor Titular Universidade Federal do Paraná).

3. BROMBERG, S.H. - Fatores morfológicos prognósticos do cancer gástrico ressecado. São Paulo, 1984. 79 pg (TESE - Mestrado - Instituto Brasileiro de Estudos e Pesquisas de Gastroenterologia).

4. CADY, B. - Lymph node metastases. Indicators but not governors of survival. Arch. Surg., 119:1067-72, 1984.

5. CAWTHORN, S.J.; GIBBS, N.M.; MARKS, C.G. - Clearance technique for the detection of lymph nodes in colorectal cancer. Am. J. Surg., 73:58-60, 1986.

6. COCHRAN, W. G. - Some methods for strenghtering the comman X2 test. Biometrics, 10:417-51, 1954.
7. COHEN, A.M.; TREMITERRA, S.; CANDELA, F; THALER, H.T.; SIGURDSON, E.R.;- Prognosis of node-positive colon cancer. Cancer, 67:1859-61, 1991.

8. COPELAND, E.M.; MILLER, L.D.; JONES, R.S. - Prognostic factors in carcinoma of the colon and rectum. Am. J. Surg. 116:875-881, 1968.

9. CUTAIT, R.- Contribuiçào ao estudo do antigeno carcinoembrionario $e$ dos antigenos de superficie ABH no câncer colo-retal. São Paulo, 1987, 138 p. (Tese - Doutorado - Faculdade de Medicina da Universidade de São Paulo).

10. DUKES, C.E. - The classification of cancer of the rectum. J.Pathol., 35:323-32, 1932.

11. DUKES, C.E. \& BUSSEY, H.J.R. - The spread of rectal cancer and its effect on prognosis. Br. J. Cancer, 12:309-20, 1958.

12. FISHER, E.R.; SAFFER, E.; FISHER, B. - Studies concerning the regional lymph node in cancer. III Response of regional lymph node cells from breast and colon cancer patients to PHA stimulation. Cancer. 30:1202-15, 1972. 
13. FISHER, E.R.; SAFFER, E.; FISHER, B. - Studies concerning the regional lymph node in cancer.VICorrelation of lymphocyte transformation of regional node cells and some histopathologic discriminants. Cancer, 32:104-11, 1973.

14. FISHER, E.R.; FISHER, B.; SAFFER, E. - Studies concerning the regional lymph node in cancer. Relationship of nodal histologic findings to citotoxicity and immunity. Arch. Pathol. Lab. Med. 101:152-5, 1977.

15. FISHER, E.R.; SWAMIDOSS, S.; LEE, C.H.; ROCKETTE, H.; REDMOND, C.; FISHER, B. - Detection and significance of occult axillary node metastases in patients with invasive breast cancer. Cancer, 42:202531, 1978.

16. GABRIEL W.B., DUKES C., BUSSEY H.J.R. - Lymphatic spread in cancer of the rectum. Br. J. Surg., 23:395$413,1935$.

17. HERRERA-ORNELAS, L.; JUSTINIANO, J.; PETRELLI. N.J.; MITTELMAN, A. - Metastases in small lymph nodes from colon cancer. Arch. Surg. 122:1253-6, 1987.

18. HOON, D.S.B.; KORN, E.L.; COCHRAN, A.J. - Variations in functional immunocompetence of individual tumor escrining lymph nodes in humans. Cancer Res., 47:1740-4, 1987.

19. HUVOS, A.G.; HUTTER, R.V.P.; BERG, J.W. - Signilicance of axillary macrometastasis and micrometastasis in mammary cancer. Ann. Surg., 173:44-6, 1971.

20. JASS, J.R. - Prognostic factors in colorectal cancer. Cur\%. Top. Pathol., 81:295-322, 1990.

21. JASS, J.R. \& MORSON, B.C. - Reporting colorectal cancer. J. Clin. Pathol., 40:1016-23, 1987.

22. JEFFERS, M.D.; Ö'DOWD, G.M.; MULCAHY, H.; STAGG, M.; O’DONOGHUE, D.P.: TONER, M. The prognostic significance of immunohistochemically detected lymph node micrometastases in colorectal carcinoma. J. Pathol., I72:183-7, 1994.

23. LEE, E.T. - Statistical methods for survival data analysis. Belmont, U.S.A., Ed. Publications Lifetime Learning, 1980. $551 \mathrm{pg}$.

24. MAMBO N.C.; GALlAGiIER H.S. - Carcinoma of the breast. The prognostic significance of extranodal extension of axillary disease. Cancer, 39:2280-5, 1977.

25. MURRAY, D.; HRENO, A.; DUTTON, J.; HAMPSON, L.G. - Prognosis in colon cancer: a pathologic reassessment. Arch. Surg., 110:908-13, 1975.

26. OHMAN, U. - Colorectal carcinoma: a survey of 1345 cases 1950-1984. Acta Chir: Scand., 151:675-9, 1985.

27. PAYNE, J.E. - International colorectal carcinoma staging and grading. Dis. Colon Rectum, 32:282-5, 1989.

28. PIHL, E.; NAIRN, R.C.; NIND, A.P.; MULLER, H.K.; HUGHES, E.S.R.; CUTHBERTSON, A.M.; ROLLO, A.J. - Correlation of regional lymph node histology with in vitro antitumor immunoreactivity in colorectal carcinoma. Cancer Res., 36:3665-71, 1976.

29. PIHL, E; NAIRN, R.C.; HUGHES, E.S.R.; CUTHBERTSON, A.M.; ROLLO, A.J. - Regional lymph node and stromal immunomorphology in colorectal carcinoma and relation to tumor spread. Pathologv, 12:15-21, 1980.

30. ROSEN, P.P.; SAIGO, P.E.; BRAUN, D.W.; WEAHERS, E.; FRACCHIA, A.A.; KINNE, D.W. - Axillary micro-and macrometastases in breast cancer: prognostic significance of tumor size. Ann. Surg., 194:585 91, 1981 .

31. SCHMITZ-MOORMANN, P.; HIMMELMANN, G.W.; BAUM, U.; NILLES, M. - Morphological predictors of survival in colorectal carcinoma: univariate and multivariate analysis. J. Cancer Res. Clin. Oncol., 113:586-92, 1987.

32. SCOTT, K.W.M, GRACE, R.II. - Detection of lymph node metastases in colorectal carcinoma before and after fat clearance. Br. J. Surg., 76:1165-7, 1989.

33. SIEGEL, S. - Estadística no paramétrica. Mexico, Ed. Trillas, 1975. $346 \mathrm{pg}$.

34. SPRATT J.R., J.S. \& SPJUT, H.J. - Prevalence and prognosis of individual clinical and pathologic variables associated with colorectal carcinoma. Cancer, 20:1976-85, 1967.

35. VAN DE VELDE, C., CARR, I. - Lymphatic invasion and metastasis. Experientia. 33:837-43, 1977.

36. WILSON, S.M., BEAHRS, O.H. - The curative treatment of carcinoma of the sigmoid, rectosigmoid and rectum. Ann. Surg., 183:556-565, 1976.

37. WILKINSON, E.J.; HAUSE, L. - Probability in lymph node sectioning. Cancer, 33:1269-74, 1974.

38. WOLMARK, N.; FISHER, E.R.; WIEAND, S.; FISHER, B. - The relationship of depth of penetration and tumor size to the number of positive nodes in Dukes C colorectal cancer. Cancer: 53:2707-12, 1984.

39. WOLMARK, N.; FISHER, E.R.; WIEAND, S. - The prognostic value of the modifications of Dukes $C$ class of colorectal cancer. Ann. Surg., 203:115-22, 1986.

Endereço para correspondência:

Dr. Sansom H. Bromberg

Av. Angélica, 589 Ap. 81

São Paulo - S. Paulo

CEP 01227-000

FAX-Fone (011) 665873

Data do recebimento: 25.08 .96

Data da revisão: 17.09 .96

Data da aprovação: 08.10 .96 